\title{
Psychometric validation of the Persian version of the Emotional Style Questionnaire
}

\author{
Nabi Nazari ${ }^{1}$ (D) Mark D. Griffiths ${ }^{2}$ \\ Accepted: 17 November 2020 / Published online: 26 November 2020 \\ (C) Springer Science+Business Media, LLC, part of Springer Nature 2020
}

\begin{abstract}
Emotions play a central role in scientific models of decision-making, human development, interpersonal processes, psychopathology, and well-being. The Emotional Style Questionnaire (ESQ) is a novel and multifaceted psychometric scale that assesses the dimensions of individual's emotional styles. The present study evaluated the validity and factor structure of the Persian ESQ. The original version of the ESQ was translated and back-translated into Persian, followed by a pilot study. A sample of university students and staff participated in a survey $(n=822)$ which included the ESQ, Overall Anxiety Severity and Impairment Scale (OASIS), World Health Organization Quality of Life Brief Version (WHOQOL-BREF), Difficulties in Emotion Regulation Scale (DERS), and Positive and Negative Affect Schedule (PANAS). Results showed that the Persian ESQ had very good internal consistency (Cronbach's alpha coefficient: 0.84 ; Composite reliability $=0.89$ ) and adequate test-retest reliability after four weeks (intraclass coefficient, $r=.71$ with $95 \% \mathrm{CI}[.63, .77])$. The confirmatory factor analysis model fitted the data well $\left(\chi^{2} /\right.$ $\mathrm{df}=2.86, \mathrm{CFI}=.970, \mathrm{SRMR}=.046, \mathrm{PCLOSE}=.85>.05, \mathrm{RMSEA}=.048,90 \% \mathrm{CI}[.043, .053])$. Also, measurement invariance indicated the ESQ had acceptable construct validity among different groups. As for criterion-related validity, the ESQ positively correlated with scores on the WHOQOL-BREF $(r=.76)$ and PANAS-positive affect $(r=.62)$, and negatively correlated with the scores on the OASIS $(r=-.68)$, DERS $(r=-.39)$, and PANAS-negative affect $(r=-72)$. The findings provide evidence that the ESQ is a reliable and valid instrument for assessing healthy emotionality among Persian speaking individuals. The Persian ESQ can be used in psychological intervention and clinical research in Iran and other Persian-speaking countries.
\end{abstract}

Keywords Emotion $\cdot$ Quality of life $\cdot$ Affective neuroscience $\cdot$ Validation $\cdot$ Well-being

\section{Introduction}

Emotions and affects play a central role in scientific models of well-being, human development, interpersonal processes, psychopathology, and decision-making (Lobue, Pérez-Edgar, \& Buss, 2019; Shackman, Fox, Lapate, \& Davidson, 2018). In well-being research, emotional well-being and life evaluation are two aspects of fundamental subjective well-being (Kahneman \& Deaton, 2010) that have received attention from a number of groups including psychiatrists and clinical psychologists (Norton \& Paulus, 2016; Rosellini \& Brown, 2019), educational psychologists (Linnenbrink-Garcia \& Pekrun,

Nabi Nazari

Nazariirani@gmail.com

1 Department of Psychology, Faculty of Human Sciences, Lorestan University, Khorramabad, Iran

2 International Gaming Research Unit, Psychology Department, Nottingham Trent University, Nottingham, NG1 4FQ, United Kingdom
2011), and other health professionals (Anthony, Krysik, \& Kelly, 2019). Emotional well-being refers to the emotional quality of an individual's daily experiences, the frequency and intensity of positive experiences (e.g., enjoyment, hope) and negative experiences (anxiety, sadness), and their affections that make life pleasant or unpleasant (Kahneman \& Deaton, 2010). Broad demographic, economic, and situational factors can affect emotional well-being such as the impact of the novel coronavirus-2019 (COVID-19) pandemic (Yang \& Ma, 2020). There is a belief that a middle way between behaviorism and cognitive science is possible in the understanding of emotional affect in the brain. Advances in neuroimaging and other technologies have enabled researchers to gain insight into what happens in the brain when individuals are exposed to emotional situations or disasters.

\section{Affect, Emotion and Emotion Regulation}

Affect denotes any experience of feeling, ranging from the simplest to the most complex feeling sensations, and 
from the normal to the most pathological emotional reactions (Barrett \& Bliss-Moreau, 2009, VandenBos \& American Psychological Association, 2015). Emotion, an affective state, refers to an event-focused, complex reaction pattern, involving experiential, behavioral, and physiological elements (Sander, 2013; VandenBos \& American Psychological Association, 2015). However, there is no absolute consensus on the definition of emotion (Izard, 2010). Emotion regulation is defined as the process of modulating one or more aspects of an emotional experience or response (Gross \& Jazaieri, 2014). It may take place either at a conscious or unconscious level. Emotion regulation refers to individuals monitoring their emotional experiences and modifying emotional expressions, deliberate behavioral change, and regulation of affective states in facing intense or unpleasant emotions (Leahy, Tirch, \& Napolitano, 2011). A number of psychometric instruments have been developed that assess emotion regulation.

\section{Emotion Assessment}

There are a number of psychometric instruments that have been used to assess different aspects of emotion. For instance, the ten-item Emotion Regulation Questionnaire (ERQ; Gross \& John, 2003) was developed to assess two central factors of emotion regulation (i.e., cognitive reappraisal and suppression). The ERQ's theoretical model is valuable in orienting researchers to regulatory strategies. However, this bi-factor framework is arguably too narrow in identifying the diversity in emotion regulation strategies, including behavioral, emotional, and cognitive patterns. The Difficulties in Emotion Regulation Scale (DERS; Gratz \& Roemer, 2004) is a multidimensional scale that assesses emotion regulation difficulties. Although a more practical and parsimonious objective instrument, DERS items predominantly relate to negative emotional states. Therefore, the DERS is not a comprehensive measure of emotion regulation (Bloch, Moran, \& Kring, 2009). Emotion regulation is viewed as a complex process, heavily depending on the context in which the emotion regulation takes place. However, these measures provide little information to address the role of the sociocultural factors that are particularly important in research on emotion regulation (Ford et al., 2015). Most of the literature on emotion regulation has focused on individual emotion (Ford et al., 2015). The lack of instruments that address the cultural and social factors involved in emotion regulation can be considered one reason for this bias in published research. A full understanding of emotion regulation requires that researchers move beyond individual emotion regulation (Mesquita, De Leersnyder, \& Albert, 2014).

\section{Cultural and Social Factors}

The debate concerning the nature of emotions has taken place in the social and cultural context of the burgeoning empirical fields of social and affective neuroscience (Kong et al., 2019; van Kleef, Cheshin, Fischer, \& Schneider, 2016). The effects of emotion regulation are viewed as a complex process, heavily dependent upon the context in which emotion regulation takes place (Aldao \& Nolen-Hoeksema, 2012). Social and cultural factors are necessary for adaptive emotional functioning (e.g., reduced pessimism, faster readjustment, increased patience, impulse control). To actualize adaptive emotional functioning, high loads of social and cultural information must be integrated and converged into fundamental emotional systems in the brain (Asma \& Gabriel, 2019). The results of emotional processing and the quality and quantity of the information eventually affect cognitive processing and shape the behaviors. Recent studies confirm the significant emotion regulation of different strategies between different cultural populations in similar conditions (Deng, An, \& Cheng, 2019; Jobson et al., 2019). Consequently, the development of an instrument that assesses emotion also needs to address the contributing social and cultural factors to gain more knowledge and comprehensive insights concerning emotion regulation.

\section{Emotional Style Questionnaire}

The recently developed Emotional Style Questionnaire (ESQ; Kesebir, Gasiorowska, Goldman, Hirshberg, \& Davidson, 2019) is a novel and multifaceted psychometric scale that assesses the dimensions of emotional style. The theoretical model underlying the ESQ is drawn from thirty years of neuroscientific studies of emotion (Davidson \& Begley, 2012). This model specifies six major dimensions of emotional life, which are relevant to psychological well-being. The ESQ dimensions were derived directly from empirical studies on the brain utilizing functional magnetic resonance imaging (fMRI) and electroencephalography (EEG) (Ricard, Lutz, \& Davidson, 2014) rather than traditional psychological methods that rely on self-report data which are prone to systematic biases and errors (for more detail, see Davidson \& Begley, 2012). For example, the use of fMRI and EEG show that resilience is marked by increased connectivity between prefrontal regulatory regions and the amygdala (Davidson \& McEwen, 2012; Kim \& Whalen, 2009). Less resilient individuals demonstrated reduced connectivity between the prefrontal cortex and amygdala than those who were more resilient. The use of fMRI and EEG allows researchers to examine whether cultural differences in emotion self-reports and behavior are also reflected in activity in specific areas of the brain related to reward, attention, and self-relevance (Tsai \& $\mathrm{Qu}, 2018)$. In the brain, the fusiform gyrus is a key structure in 
a circuit known to be important for social intuition. While looking at pictures of faces, individuals high on social intuition displayed high levels of fusiform gyrus activation and low-to-moderate amygdala activity, a pattern that is reversed for individuals at the low end of this dimension (Dalton et al., 2005). The ESQ's six dimensions are outlook, resilience, social intuition, self-awareness, sensitivity to context, and attention (explained in more detail in the next section). Each of these dimensions describes a continuum with two extremes (i.e., high and low). Generally, each dimension reflects increased or reduced activity in the brain circuits that underlie these dimensions.

\section{The Six Dimensions of ESQ}

Outlook refers to the ability for individuals to maintain positive emotion across time. The pleasant feelings of positive emotion, experienced by individuals with high outlook, persist for a longer duration than those with low outlook (Margolis \& Lyubomirsky, 2018). Those with a positive emotional outlook appear to have a greater tendency to undertake healthy behaviors (Fredrickson \& Joiner, 2018; Pressman, Jenkins, \& Moskowitz, 2019), and have a higher medication adherence (Millstein et al., 2016). Applying a neuro-affective lens, a higher ability to savor positive emotions can promote well-being (Davidson, 2015).

Resilience refers to effective (i.e., positive) adaptation to adversity (Smith \& Hanni, 2019). Resilience is defined as a personal resource that enhances individual adaptation and positive personality characteristics (Helmreich et al., 2017) and that promotes adaptive emotional responses in the face of adversity and enhance adoption (Wang, Goldenberg, Dorison et al., 2020). More specifically, in an unexpected crisis, resilience refers to the individual ability to continue functioning and readjustment (Bryce, Ring, Ashby, \& Wardman, 2020). Like the outlook dimension, resilience describes a quality of affective chronometry or the temporal dynamics of emotional responding. While outlook refers to the ability to maintain positive emotion, resilience refers to the ability to bounce back from negative emotion (Schuyler et al., 2014). Beyond recovery from negative affect, sustained responses or short-lived responses to positive experiences are associated with psychopathology (Lapate \& Heller, 2020) and/or depressive symptoms (Lapate et al., 2014). Persistence of the positive emotion can be an effective mechanism to reduce the risk of depression in traumatic situations in which individuals' resilient abilities are critical (Fredrickson, Tugade, Waugh, \& Larkin, 2003; Laird, Krause, Funes, \& Lavretsky, 2019). Consequentially, the ability to bounce back quickly from negative events inevitably facilitates the maintenance of positive affect and the ability to maintain positive affect inevitably facilitates bouncing back from negative events.
Self-awareness refers to the ability to perceive one's bodily signals that reflect emotions. Although individuals respond to stressful situations in a variety of ways, increasing knowledge and developing skills can lead to effective prevention. Some individuals are accurate in perceiving internal bodily cues, while others are less accurate. Bodily sensations are usually associated with high anxiety. These individual differences in awareness of internal bodily states are likely to play an essential role in various aspects of modulating emotional responses (Ardelt \& Grunwald, 2018; Oser et al., 2019).

Sensitivity to context refers to whether an emotional and behavioral response matches the provided social cue. Social cognition and behavior rely on sensitivity to intention and context (Aldao, 2013). Sensitivity to context is a prerequisite for social interaction and learning. This dimension can be considered as the outer-directed version of self-awareness. Whereas self-awareness reflects attunement to one's own physiological and emotional cues, sensitivity to context reflects attunement to the social environment. For instance, individuals with better awareness skills demonstrate more effective self-regulatory ability in social interaction (Ferri, Chiarelli, Merla, Gallese, \& Costantini, 2013). Sensitivity to emotional context is an emerging construct for characterizing adaptive or maladaptive emotion regulation, but few measurement approaches exist (Myruski, Bonanno, Gulyayeva, Egan, \& Dennis-Tiwary, 2017).

Social intuition refers to individuals' ability to make a rapid judgements and attunement to nonverbal social signals (e.g., facial expressions, body language, and gesture). Individuals high on the social intuition dimension are adept at reading nonverbal cues and on decoding the motives and intentions based on certain cues (Norman \& Price, 2012). Highly socially intuitive individuals may effectively manage their emotions and the impact of emotions in their interactions, and also infer social information from others' emotional states (Armstrong, van der Heijden, \& Sadler-Smith, 2012). Individuals with autism have social impairments and lack social intuition. Being extremely low on the social intuition dimension includes individuals on the autism spectrum, and who struggle to read non-verbal cues (Kret, Sinke, \& de Gelder, 2011).

Attention refers to the ability to screen out distractions and stay focused. Individuals with lower attentional skills may experience a high attentional bias to potential sources of threat (Hagerty \& Williams, 2020). Experimental work shows that emotion plays an essential role in concentrating or diffusing attention (Dolcos et al., 2020). The ability to resist emotional distractions and impulses enables individuals to concentrate on goals and recover from emotionally-demanding tasks (Restubog, Ocampo, \& Wang, 2020). Intentional selective attention to positive information reflects emotion regulation, and regulating attention is a critical component in behaving adaptively. Attention and emotion are intimate partners, and higher degrees of attention are associated with higher 
emotional balance and good well-being (Killingsworth \& Gilbert, 2010).

This investigation of individual differences in affective chronometry has the potential to explain why specific individuals are vulnerable to affective disorders and why others are resilient, as well as why some individuals modulate their emotional responses in context-appropriate ways, and some do not.

\section{The Present Study}

The optimal dimensions of individuals' emotional wellness need to be mapped out, particularly in the current global health crisis (Holmes et al., 2020). Understanding the effective coping styles in stressful situations is of paramount importance (Folkman, 2010; Folkman \& Moskowitz, 2004). The present study was carried out because it is necessary to validate a scale for emotional health among Persian populations, particularly in the context of the coronavirus disease-2019 (COVID-19) pandemic given the high numbers of infections and deaths in Iran (where the present study was carried out). The present study was designed to evaluate the validity and factor structure of the Persian ESQ. It was expected that high scores on the ESQ would be positively correlated with quality of life and positive affect, and negatively correlated with difficulties in emotion regulation, negative affect, and anxiety.

\section{Method}

\section{Participants}

The sample comprised 822 participants (431 males and 391 females) aged between 18 and 56 years (mean age 31.46 years; $\mathrm{SD}=8.74)$. The demographic characteristics of the sample are shown in Table 1. The inclusion criteria were being over the age of 18 years and fluency in the Persian language.

\section{Measures}

Emotional Style Questionnaire (ESQ; Kesebir et al., 2019). The ESQ is a 24-item self-report measure developed to assess emotional health comprising six dimensions including outlook (e.g., "I am very good at seeing the positive side of things"), resilience (e.g., "When I experience a setback, I do not stay upset for very long"), social intuition (e.g., "When I am talking with people, I am always attuned to their emotional state"), self-awareness (e.g., "Usually, I am not attentive to what is going on in my body"), sensitivity to context (e.g., "I have sometimes been told that I behaved in a socially inappropriate way"), and attention (e.g., "I do not get distracted
Table 1 Demographic characteristics of the sample $(N=822)$

\begin{tabular}{llll}
\hline Item & Value & Test & $p$ \\
\hline $\begin{array}{l}\text { Categorical variables } \\
\text { Gender, } n \text { (\%) }\end{array}$ & & & \\
$\quad$ Women & $391(47.6)$ & $\chi^{2}=1.95$ & .16 \\
$\quad$ Man & $431(52.4)$ & & \\
Marital, $n$ (\%) & & & \\
Single & $385(46.8)$ & $\chi^{2}=3.29$ & .07 \\
In Relationship & $437(53.2)$ & & \\
Continues variables $M(S D)$ & & & \\
Age & $31.46(8.74)$ & & \\
Outlook & $2.95(1.48)$ & & \\
Resilience & $3.44(1.57)$ & \\
Social intuition & $3.66(1.48)$ & \\
Self-awareness & $3.41(1.49)$ & \\
Sensitivity context & $3.331(1.21)$ & \\
Attention & $3.57(1.43)$ & \\
healthy emotionality average & $3.39(.69)$ & \\
healthy emotionality & $81.54(16.69)$ &
\end{tabular}

Note: $n=$ frequency; $\mathrm{y}=$ years; $\mathrm{M}=$ mean; $\mathrm{SD}=$ standard deviation; $\mathrm{ESQ}=$ Emotional Style Questionnaire; WHOQOL-BREF=World Health Organization Quality of Life Brief Version; DERS = Difficulties In Emotion Regulation Scale; OASIS=Overall Anxiety Severity and Impairment Scale; PANAS=Positive and Negative Affect Schedule. $\mathrm{PA}=$ positive affect $\mathrm{NA}=$ negative affect

easily, even in situations where a lot is going on") (four items in each dimension). Response options range from 1 (strongly disagree) to 7 (strongly agree) on a Likert scale with total scores ranging from 24 to 168 (see Table 2 for a brief interpretation of obtained score in each dimension of the ESQ). Each of these dimensions describes a continuum with two extremes (i.e., high and low). The overall ESQ score can be considered as a proxy for individual healthy emotionality and refers to the overall individuals' strengths in adaptive emotional functioning. Psychometric properties of the scale are presented in the Results section.

Overall Anxiety Severity and Impairment Scale (OASIS; Norman, Cissell, Means-Christensen, \& Stein, 2006). The OASIS is a unidimensional transdiagnostic five-item self-report measure that assesses anxiety symptoms and their severity and impairment over the over the past week (e.g., "how often did you avoid situations, places, objects, or activities because of anxiety or fear? "). Response options range from 0 (never) to 4 (all the time) with total scores ranging from 0 to 20. Higher scores indicate higher anxiety. Internal reliability in the present study was very good (Cronbach's $\alpha=0.82$ ).

World Health Organization Quality of Life Brief Version (WHOQOL-BREF; WHO QOL Group, 1998; Skevington et al., 2004). The WHOQOL-BREF is a 26-item self-report scale that assesses quality of life across four domains including physical (e.g., "How satisfied are you with your sleep?"), 
Table 2 Brief interpretation of obtained score in each dimension of the ESQ

\begin{tabular}{|c|c|c|}
\hline Dimension & High score & Low score \\
\hline Outlook & $\begin{array}{l}\text { Individuals high on this dimension are generally upbeat } \\
\text { and good at seeing the silver lining in every cloud. }\end{array}$ & $\begin{array}{l}\text { Individuals low on this dimension may experience joy, } \\
\text { but their joys dissipate fast. They have a tendency } \\
\text { towards gloominess and pessimism. }\end{array}$ \\
\hline Resilience & $\begin{array}{l}\text { Individuals high on this dimension can recover from } \\
\text { setbacks and bounce back from challenges relatively } \\
\text { easily and rapidly. }\end{array}$ & $\begin{array}{l}\text { Individuals low on this dimension are slow to recover } \\
\text { from adversity, and are often crippled by it. }\end{array}$ \\
\hline Sensitivity to context & $\begin{array}{l}\text { Individuals high on this dimension know how to modify } \\
\text { their responses to the implicit rules and expectations } \\
\text { that govern different social situations. }\end{array}$ & $\begin{array}{l}\text { Individuals low on this dimension are at times } \\
\text { insufficiently sensitive to the surrounding context and } \\
\text { their behavior can be judged as inappropriate by } \\
\text { others. }\end{array}$ \\
\hline Social Intuition & $\begin{array}{l}\text { Individuals high on this dimension are acutely sensitive } \\
\text { to the emotional states of others. }\end{array}$ & $\begin{array}{l}\text { Individuals low on this dimension, have a difficult time } \\
\text { reading other individuals' emotions. }\end{array}$ \\
\hline Self-awareness & $\begin{array}{l}\text { Individuals high on this dimension are conscious of their } \\
\text { thoughts and feelings and are attuned to the messages } \\
\text { that their body sends them. }\end{array}$ & $\begin{array}{l}\text { Individuals low on this dimension are less sensitive to } \\
\text { their internal signals and may experience confusion } \\
\text { about the nature of their emotions. }\end{array}$ \\
\hline Attention & $\begin{array}{l}\text { Individuals high on this dimension have a sharp and clear } \\
\text { focus. }\end{array}$ & $\begin{array}{l}\text { Individuals low on this dimension get easily captured by } \\
\text { other attention-grabbing stimuli in the environment. }\end{array}$ \\
\hline
\end{tabular}

psychological (e.g., "Are you able to accept your bodily appearance?"), social (e.g., "How satisfied are you with your personal relationships?"), and environmental (e.g., "How satisfied are you with your transport?"). Of the 26 items (which includes two general items), 24 items generate a profile and score for each of the four domains of QOL (Skevington et al., 2004). Response options range from 1 (not at all) to 5 (completely) with total scores ranging from 24 to 120 . Higher scores on the subscales and total scores indicate better quality of life. Internal consistency in the present study was very good $(\alpha=.88)$.

Difficulties in Emotion Regulation Scale (DERS; Gratz \& Roemer, 2004). The DERS is a 36-item self-report scale that assesses overall difficulties in emotion regulation. The DERS comprises six subscales: no acceptance of emotional responses, difficulties engaging in goal-directed behavior, impulse control difficulties, lack of emotional awareness, limited access, and lack of emotional clarity. Participants rate their emotional state on a scale from 1 (almost never) to 5 (almost always) with total score ranging from 36 to 180 . Higher scores indicate greater impairment. Internal reliability in the present study was excellent $(\alpha=.90)$.

The Positive and Negative Affect Schedule (PANAS; Watson, Clark, \& Tellegen, 1988). The PANAS is a brief 20-item self-report scale that assesses positive affect (PA; ten items; e.g., Interested) and negative affect (NA; ten items; e.g., Nervous) (Watson et al., 1988). Each item is rated on a five-point scale from 1 (very slightly) to 5 (extremely) describe different feelings and emotions over the past month. The total score ranges from 10 to 50 in each sub-scale. Higher scores indicate greater positive or negative mood experienced in the past week. Internal reliability in the present study was very $\operatorname{good}(\alpha=.87)$.

\section{Procedure and Ethics}

Ethics Ethics approval was granted by the research team's Institutional Review Board (IRB). The IRB reviewed the research protocol to ensure participant confidentiality, sampling, and obtaining informed consent. The permission was granted before data collection from the first author of the original ESQ. Participants provided informed consent and were ensured anonymity and confidentiality and were explicitly asked not to provide their names or other personal identification information. They were notified that participation was voluntary and that they had the right to withdraw their participation at any time.

Development of the Scale The guidelines proposed by Beaton, Bombardier, Guillemin, and Ferraz (2000) were utilized for the transcultural adaptation and content validity of the ESQ. Initially, two Persian translators (one knowledgeable of the scale concepts, and one not knowledgeable) independently translated the ESQ to Persian. An expert committee evaluated both translated versions to resolve any discrepancies and synthesize a consensual version. Two native English translators then created two backward translation of the consensual version. The expert committee, translators, and research team reviewed all reports to reach the produce prefinal scale.

In the final stage, a pilot study was performed with 35 participants, selected from the target population, to verify the feasibility of the ESQ. The participant debriefing was performed to find actual and potential items' ambiguity or errors (linguistic, grammar). For the pilot study itself, an invitation letter was prepared to explain the study aim and to request a review of translated ESQ version. The participants were 
encouraged to provide feedback. According to received feedback, the translated ESQ items were easily understandable. The median response time was $15-20 \mathrm{~min}$.

Sampling The participants for the main study were recruited from university students and staff via individuals' registered emails. First, a convenience sample of eight universities was selected from the university list in Iran. The Heads of Offices of Research at the universities were then informed about the study and were asked if they would participate. Three universities accepted the invitation to participate. A survey letter was sent to the research chancellors. A list of potential participants was randomly selected to take part in the study via random generator. Over $30 \%$ of participants recruited in the first seven weeks, $60 \%$ by Week 11 , and the remainder by Week 16 . Data collection (including test-retest) began in the first week of February 2020 and ended in the third week of June 2020. Overall, the study was conducted during the COVID-19 outbreak.

Sample Size A graded scale of sample sizes for scale development considers 500 to be very good, and over 1000 to be excellent (Comrey \& Lee, 2013). The study sample size in the present study $(N=822)$ was therefore more than acceptable.

\section{Data Analysis}

There were no missing values in the assessed variables, and therefore no imputation method was implemented. Distributions of frequency, chi-square tests, and independent $t$-tests (where suitable) were conducted to test the difference between groups. Univariate normality was tested by skewness and kurtosis levels. Item analysis was performed by means and standard deviations. A maximum-likelihood confirmatory factor analysis (CFA) was conducted on the hypothesized (six-factor) model for the total sample $(n=822)$ using AMOS 24. The following values demonstrate an excellent fitting model (Hu \& Bentler, 1999): $1<\chi^{2} / d f<3$, comparative fit index $(\mathrm{CFI})>0.95$, root mean square error of approximation (RMSEA) $<0.06$, and standardized root mean square residual $(\mathrm{SRMR})<0.06$. To further examine the changes in the model fitting index, measurement invariance was tested within the framework of multi-group CFA (Byrne, 2016; Kline, 2016). Measurement invariance is important to assess the psychometric equivalence of a construct across different groups. A related research question that requires a different CFA approach is: "Does the scale perform differently for one population than another?"(Schumacker \& Lomax, 2016; Byrne, 2016). The total sample divided two groups regarding gender. First, the six-factor model for the groups (male, female) was evaluated. Given the model fitted well for both groups (see Results), measurement invariance was performed. This included configural invariance, metric invariance, scalar invariance, and error variance invariance across gender for the ESQ. A change in CFA $(\triangle C F I)<.01)$, a change TLI $(\triangle T L I)$ $<.01$, and a change in RMSEA $(\triangle \mathrm{RMSEA})<.015$ indicate a non-invariance between groups (Chen, 2007; Cheung \& Rensvold, 2002).

A Cronbach's alpha reliability coefficient was generated to assess internal consistency. An intraclass correlation coefficient (ICC) was used to evaluate test-retest reliability. The composite reliability (CR) was utilized for satisfactory reliability. Also, McDonald's construct validity was generated for each subscale. The Cronbach alpha if item deleted values and corrected item correlation values are shown in Table 4 . The test-retest was evaluated by ICC after four weeks $(N=189)$. The present study considered as acceptable Cronbach alpha $>0.7, \mathrm{CR}>0.8$, and the test-retest reliability $>0.7$. Convergent validity was tested utilizing average extracted variance (AVE). An AVE of .5 or higher is a good rule of thumb, suggesting adequate convergence (Henseler, Hubona, \& Ray, 2016).

Criterion-related validity of the ESQ was tested by examining the (Pearson) correlations with quality of life (WHOQOL-BREF), and difficulties in emotion regulation (DERS), positive and negative affect (PANAS), and anxiety (OASIS). The measures were selected to tap both eudemonic positive aspects (e.g., positive affect) and negative aspects (anxiety) of psychological well-being. The magnitude of Pearson correlations $(r)$ varies around $.10, .30$, and .50 correspond to for small, medium, and large the Cohen's $d$ effect sizes, respectively (Cohen, 1992). In addition, the coefficient of determination $\left(r^{2}\right)$ was calculated to express the proportion of variation in the criterion that is accounted for by the target measure.

\section{Results}

The demographic characteristics of the sample were illustrated in Table 1. The univariate normality of the data was checked. Values of skewness and kurtosis for all 24 items were within $<|1|$. The VIF for the 24 items were in acceptable range (see Table 3). Males obtained statistically significant higher scores according to independent $\mathrm{t}$-test for the total ESQ $(t[820]=$ 2.06, $p=.039$, Cohen's $d=.15$ ), outlook dimension of the ESQ $(t[820]=2.59, p=.01$, Cohen's $d=.18)$, and positive affect $(t[820]=2.71, p=.01$, Cohen's $d=.19)$. Also, for negative affect, male scores were statistically significantly lower than females $(t[820]=2.46, p=.01$, Cohen's $d=.16)$. Using one-way ANOVAs, the findings showed that males scored significantly higher than females on outlook $(F[1,820]=$ $4.26, p=.03)$, ESQ $(F[1,820]=6.73, p=.01)$, and positive affect $(F[1,820]=19.72, p<.01)$. Females scored significantly higher than males on negative affect $(F[1,820]=6.07$, $p=.01$ ). Gender differences for all other ESQ dimensions and other variables were non-significant. 
Table 3 Item statistics

\begin{tabular}{|c|c|c|c|c|c|c|}
\hline Item Number & Dimension & Factor Loading & Skewness & Kurtosis & $\begin{array}{l}\text { Squared Multiple } \\
\text { Correlation }\end{array}$ & $\begin{array}{l}\text { Cronbach's } \\
\text { If item deleted }\end{array}$ \\
\hline 1 & Outlook & .893 & .18 & -.32 & .785 & .825 \\
\hline 7 & Outlook & .914 & .25 & -.60 & .794 & .827 \\
\hline 13 & Outlook & .905 & .36 & -.70 & .811 & .827 \\
\hline 19 & Outlook & .902 & .17 & -.88 & .775 & .829 \\
\hline 2 & Resilience & .916 & .35 & -.88 & .735 & .825 \\
\hline 8 & Resilience & .888 & .17 & -1.05 & .740 & .826 \\
\hline 14 & Resilience & .913 & .31 & -1.02 & .809 & .826 \\
\hline 20 & Resilience & .892 & .36 & -.93 & .773 & .827 \\
\hline 3 & Social Intuition & .913 & .08 & -.78 & .556 & .832 \\
\hline 9 & Social Intuition & .887 & .17 & -.81 & .619 & .834 \\
\hline 15 & Social Intuition & .894 & .10 & -.93 & .672 & .833 \\
\hline 21 & Social Intuition & .770 & .35 & -.98 & .449 & .837 \\
\hline 4 & Self-Awareness & .811 & .02 & -.25 & .571 & .830 \\
\hline 10 & Self-Awareness & .874 & .27 & -.39 & .731 & .831 \\
\hline 16 & Self-Awareness & .899 & .25 & -.54 & .751 & .834 \\
\hline 22 & Self-Awareness & .838 & .29 & -.20 & .566 & .837 \\
\hline 5 & Sensitivity to Context & .899 & .15 & -1.12 & .650 & .831 \\
\hline 11 & Sensitivity to Context & .904 & .16 & -.99 & .689 & .828 \\
\hline 17 & Sensitivity to Context & .779 & .15 & -1.14 & .541 & .828 \\
\hline 23 & Sensitivity to Context & .844 & .12 & -1.15 & .531 & .826 \\
\hline 6 & Attention & .889 & .40 & -.65 & .652 & .830 \\
\hline 12 & Attention & .841 & .60 & -.57 & .539 & .827 \\
\hline 18 & Attention & .900 & .55 & -.72 & .728 & .828 \\
\hline 24 & Attention & .863 & .59 & -.62 & 679 & .828 \\
\hline
\end{tabular}

Note: Emotional Style Questionnaire is provided in the supplementary data

Maximum likelihood $(M L)$ CFA was robust according to generated values (skewness $<2$ and kurtosis $<7$ ), in the normality test (Finney \& DiStefano, 2013). The CFA produced a good fit for the model comprising six first-order factors representing each emotional style dimension for the total sample $(n=822)\left(\chi^{2} / \mathrm{df}=2.86, \mathrm{CFI}=.970\right.$, $\mathrm{SRMR}=.046, \mathrm{PCLOSE}=.85>.05, \mathrm{RMSEA}=.048,90 \%$ CI $[.043, .053])$. Maximum-likelihood CFA for male $(n=$ $431)$ sample yielded a good fit of the model $\left(\chi^{2} / \mathrm{df}=2.22\right.$, $\mathrm{CFI}=.963, \mathrm{SRMR}=.046, \mathrm{PCLOSE}=.19>.05$, RMSEA $=.053,90 \%$ CI $[.047, .059])$. The CFA for females $(n=371)$ sample also indicated the model fitted well to the data $\left(\chi^{2} / \mathrm{df}=2.01, \mathrm{CFI}=.966, \mathrm{SRMR}=.044\right.$, PCLOSE $=.41>.05$, RMSEA $=.036,90 \%$ CI [.044, .057]). Measurement invariance was performed across gender, including configural invariance, metric invariance, scalar invariance, and error variance invariance. $(\triangle T$ LI $<.01, \triangle C F I<.01$; Table 3$)$.

The Cronbach alpha of the ESQ indicated very good internal reliability $(\alpha=0.84)$. The Cronbach alpha if item deleted values and corrected item correlation values are shown in Table 4. ICC evaluated the test-retest. After four weeks of the original validation study, the survey was re-sent to approximately half of the participants $(n=400)$ who were randomly selected by the random number generator. Of these, 189 surveys were returned. In terms of consistency, the ICC was .71 with $95 \%$ CI $[.63, .77]$ for the single measure.
The sub-scale validity is shown in Table 6. The Cronbach alpha coefficients and composite reliability, as well as McDonald's coefficient omega $(\omega)$ were generated for each dimension and all the values were satisfactory ( $\alpha$ [.81-.90]; CR [.87-.91]; $\omega$ [.90-.94]). Convergent validity of the ESQ was tested utilizing average extracted variance (AVE) and was .74. Also, AVE was calculated for each dimension ranging between .64-.75. The six dimensions of the ESQ scores correlated with the other measures (see Table 5). As for criterion-related validity, the ESQ score correlated with the score on WHOQOL$\operatorname{BREF}(r=.76,95 \% \mathrm{CI}[.72, .78], p<.01)$; the OASIS $(r=-68$, $95 \%$ CI $[-.71,-.64], p<.01)$, DERS $(r=-.39,95 \%$ CI $[-.45$, $-.33], p<.01)$, PANAS: PA $(r=.62,95 \% \mathrm{CI}[.58, .66], p<.01$, and NA $(r=-72,95 \%$ CI $[-.76,-.69], p<.01)$ (see Table 6).

\section{Discussion}

A healthy emotional life is a critical determinant of good psychological well-being. The present study was designed to evaluate the validity and factor structure of the Persian version of the Emotional Style Questionnaire (ESQ). The results of the study demonstrated that the ESQ is a valid and reliable instrument that assesses healthy emotionality among the Persian-speaking Iranian population. The evaluation of internal consistency, tested by Cronbach alpha, composite reliability, and McDonald's construct reliability, all demonstrated that the Persian ESQ had 
satisfactory reliability. The results also confirmed that the ESQ had a six-factor structure, and the findings were consistent with the original psychometric validation study (Kesebir et al., 2019). Measurement invariance analysis indicated that the sixfactor ESQ had acceptable invariance in relation to the gender aspects of configural, metric, scalar, and error variance.

In contrary to the original study (Kesebir et al., 2019), females in the present study obtained lower scores on the ESQ than males. Also, females obtained lower scores on the outlook dimension of the ESQ and in positive affect than males. Moreover, females' scores on negative affect were significantly higher than males. The study was carried out during the initial COVID-19 outbreak and there is recent evidence that females report more stress than males during the COVID-19 pandemic, both among healthcare staff (Badahdah, Khamis, \& Mahyijari, 2020) and the general population (Limcaoco, Mateos, Fernandez, \& Roncero, 2020). Generally, females experience more vulnerability to stress, as well as an increased risk for developing mental disorders during stressful life events compared to males (Tolin \& Foa, 2008).

To evaluate criterion-related validity, convergent validity, and discriminant validity, both the ESQ and its six dimensions were positively correlated with measures for quality of life (WHOQOL-BREF) and positive affect (PANAS-PA), and negatively correlated with difficulties in emotion regulation (DERS), negative affect (PANAS-NA), and anxiety (OASIS). These correlations were all as expected given that the ESQ assesses adaptive emotional functioning quality. Individual differences in abilities to implement emotion regulation strategies undoubtedly exist and may be relevant to anxious individuals' emotion regulation difficulties. Adaptive emotional functioning is critical for emotional well-being. Also, the six dimensions are relevant in identifying individuals' strengths and weaknesses in their emotional functioning. For instance, individuals with different mental disorders demonstrate a similar levels of impairment in emotion regulation, including limited coping skills, a tendency to utilize more maladaptive emotional functioning, reduced resilience, and a reduced sensitivity to contexts (Anderson et al., 2018; Lavender et al., 2015). In the present study, higher outlook and resilience were associated with higher quality of life scores on the WHOQOL-BREF. In relation to anxiety, there is evidence that individuals with anxiety disorders rely more on maladaptive strategies and less on adaptive strategies. They also report wide-ranging difficulties related to emotional experience and emotion regulation (Gross \& Jazaieri, 2014). In this sense, the overall score on ESQ can be considered a standalone measure of healthy emotionality. Healthy emotionality is a proxy representing the weakness and strength of adaptive emotion functioning as well as individual differences in affective chronometry. Researchers using the ESQ can use it to evaluate individual differences in emotional styles and it has the potential to explain why specific individuals are vulnerable to affective disorders and why others are resilient, as well as why some individuals modulate their emotional responses in context-appropriate ways, and some do not. Overall, the moderate to high correlations between the ESQ dimensions and the aforementioned scales indicate the instrument has acceptable criterion-related validity, convergent validity, and discriminant validity.

\section{Future Implications}

To enhance effectiveness of psychological interventions, the ESQ addresses the need to mechanistically target factors which are both causally associated with poor mental health and modifiable by an intervention. Poorer coping strategy and emotional dysregulation are the powerful predictors of threatening behaviours and suicidal ideation (Ong \& Thompson, 2019). Understanding how individuals adapt to threats to well-being may provide new insight about the common targeted mechanisms of well-being. For example, individuals with higher outlook and optimistic attitudes experience low levels of depression (Lemola, Räikkönen, Gomez, \& Allemand, 2013). Application of resilience in education can reduce teachers' emotional labor and enhance teachers' socioemotional competencies. Resilience is very relevant to the adversity experienced by many individuals during the COVID-19 pandemic (Li, Ghosh, \& Nachmias, 2020). Facilitating psychological resilience for vulnerable groups (e.g., healthcare staff, individuals with a mental disorder), particularly those assigned to the front lines of the COVID-19 crisis, is arguably one of the highest priorities during the pandemic (Santarone, McKenney, \& Elkbuli, 2020).

Table 4 Invariance measurement $(\mathrm{N}=822)$

\begin{tabular}{|c|c|c|c|c|c|c|c|}
\hline Type of invariance & $\begin{array}{l}\chi^{2 /} \\
d f\end{array}$ & CFI & $\Delta \mathrm{CFI}$ & TLI & $\Delta \mathrm{TLI}$ & AIC & RMSEA $90 \%[\mathrm{CI}]$ \\
\hline Configural & 2.13 & .968 & - & .963 & - & 1283.14 & $.034[.031, .038]$ \\
\hline Gender weak (Metric) & 1.97 & .967 & .001 & .965 & .002 & 1264.55 & $.034[.031, .037]$ \\
\hline Gender strong (Scalar) & 1.94 & .967 & .001 & .966 & .003 & 1238.27 & $.033[.030, .036]$ \\
\hline Gender Strict & 1.83 & .967 & .001 & .968 & .006 & 1210.13 & $.032[.029, .025]$ \\
\hline
\end{tabular}

Note CFI = comparative fit index; TLI = Tucker-Lewis index; RMSEA = root mean square error of approximation $(\mathrm{RMSEA})$; CI = confidence interval 
Table 5 Validity analysis of the ESQ dimensions

\begin{tabular}{|c|c|c|c|c|c|c|c|c|c|c|c|}
\hline Dimension & Factor Loading & CR & AVE & $\alpha$ & $\omega$ & ESQ & NA & PA & OASIS & DERS & QOL \\
\hline Outlook & .90 & .92 & .74 & .94 & .94 & $.54 * *$ & $-.37^{* *}$ & $.34^{* *}$ & $-.34^{* *}$ & $-.15^{* *}$ & $.43^{* *}$ \\
\hline Resilience & .89 & .92 & .75 & .936 & .94 & $.54 * *$ & $-.40^{* *}$ & $.36^{* *}$ & $-.37^{* *}$ & $-.17^{* * *}$ & $.43^{* *}$ \\
\hline Sensitivity to Context & .84 & .87 & .64 & .883 & .91 & $.53 * *$ & $-.32^{* *}$ & $.28^{* * *}$ & $-.31^{* *}$ & $-.20^{* *}$ & $.32^{* * *}$ \\
\hline Social Intuition & .83 & .90 & .71 & .897 & .92 & $.46^{* *}$ & $-.25^{* *}$ & $.21^{* * *}$ & $-.26^{* *}$ & $-.17^{* * *}$ & $.29^{* * *}$ \\
\hline Self-awareness & .82 & .90 & .70 & .896 & .91 & $.45 * *$ & $-.39^{* *}$ & $.32^{* *}$ & $-.34^{* *}$ & $-.25^{* *}$ & $.37^{* *}$ \\
\hline Attention & .81 & .88 & .65 & .898 & .90 & $.47 * *$ & $-.36^{* *}$ & $.29^{* *}$ & $-.34^{* *}$ & $-.19^{* *}$ & $.33^{* *}$ \\
\hline
\end{tabular}

Note: $\mathrm{CR}=$ composite reliability; $\mathrm{AVE}=$ average variance extracted; $\mathrm{MaxR}(\mathrm{h})=\mathrm{McDonald}$ construct reliability; $\alpha=$ Cronbach alpha coefficient; WHOQOL-BREF=World Health Organization Quality of Life Brief Version; DERS = Difficulties In Emotion Regulation Scale; OASIS=Overall Anxiety Severity and Impairment Scale; PA = positive affect; NA = negative affect;

$\omega=$ McDonald's coefficient omega

$* *$ Correlation is significant at the $p<.01$ level (2-tailed)

Self-awareness can facilitate treatment from trauma and find productive solutions in event-based traumatic situations (Nazari, Aligholipour \& Sadeghi, 2020). Clinical research has suggested health benefits of body awareness for patients with a variety of diagnoses. Emotional well-being can be associated with preventive behaviors. For instance, one study found that girls with higher healthy emotionality are $70 \%$ less likely to smoke cigarettes than their peers (Scal, Ireland, \& Borowsky, 2003). Another study found that adults with positive emotions are $36 \%$ less likely to develop mobility problems than their peers (Ostir, Markides, Black, \& Goodwin, 2000).

The ESQ six dimensions can be promoted via welldocumented interventions. For example, emotional wellbeing can be enhanced by transdiagnostic intervention (Barlow et al., 2017; Wilner Tirpak et al., 2019). Outlook can be promoted by positive psychology interventions which aim to improve QOL, life satisfaction, optimistic perspective, and savoring (Phillips, 2018; Steinhauser, Maier, \& Steinhauser, 2017). Psychological resilience can be promoted

Table 6 Correlations between the ESQ and other Measures $(\mathrm{N}=822)$

\begin{tabular}{llllllll}
\hline Measure & $\alpha$ & Mean & SD & \multicolumn{2}{l}{ Correlations } \\
\cline { 3 - 7 } & & & & $r$ & $95 \% \mathrm{CI}$ & $r^{2}$ \\
\hline WHOQOL-BREF & .88 & 44.52 & 6.85 & $.76^{* *}$ & {$[.72, .78]$} & .58 \\
DERS & .90 & 66.82 & 14.02 & $-.39 * *$ & {$[-.45,-.33]$} & .16 \\
OASIS & .82 & 8.96 & 2.52 & $-.68^{* *}$ & {$[-.71,-.64]$} & .46 \\
PANAS PA & .87 & 19.72 & 5.14 & $.62^{* *}$ & {$[.58, .66]$} & .38 \\
PANAS NA & .89 & 24.35 & 3.98 & $-.72 * *$ & {$[-.76,-.69]$} & .52 \\
\hline
\end{tabular}

Note. WHOQOL-BREF=World Health Organization Quality of Life Brief Version; DERS = Difficulties In Emotion Regulation Scale; OASIS=Overall Anxiety Severity and Impairment Scale; PANAS=Positive and Negative Affect Schedule. PA = positive affect; $\mathrm{NA}=$ negative affect; $\alpha=$ Cronbach alpha coefficient; $r^{2}=$ coefficient of determination

$* *$ Correlation is significant at the $p<.01$ level (2-tailed) by interventions which aid recovery from negative emotion and prevent mental problems (Helmreich et al., 2017). Selfawareness can be enhanced by mindfulness therapy or transdiagnostic treatment (Gross, Uusberg, \& Uusberg, 2019; Chin, Slutsky, Raye, \& Creswell, 2019). Attention can be targeted by emotion regulation and cognitive behavioral therapies (Renna, Quintero, Fresco, \& Mennin, 2017). Several studies suggest that mindfulness meditation training can successfully increase attentional skills (e.g., Mrazek, Franklin, Phillips, Baird, \& Schooler, 2013; Lutz, Slagter, Dunne, \& Davidson, 2008). Cognitive therapy and meditation training can promote social characteristics and well-being (Davidson \& McEwen, 2012).

The ESQ offers promise as a tool for better understanding the psychopathology associated with stressful situations (e.g., global disasters) and for identifying individuals in need of mental health services. Psychological preparedness for global disasters is generally classified in two domains: (i) cognitive aspects directed at the threat, and (ii) affective aspects (Every, McLennan, Reynolds, \& Trigg, 2019; Roudini, Khankeh, \& Witruk, 2017). For both domains, the Emotional Style model specifies six major dimensions of emotional life, which are each prominently relevant to cognitive aspects (e.g., knowledge of the contextual factors, sensitivity to context, social intuition, attention), affective aspects (e.g., self-awareness, understanding emotional consequences, emotion regulation), and psychological well-being (e.g., outlook, resiliency).

\section{Limitations}

The findings of the present study should be interpreted in light of several limitations. The data were collected during the initial COVID-19 outbreak which likely negatively affected the emotional well-being and the other study psychological constructs. Also, to minimize infection risk via traditional survey method, online data collection was utilized. Online data collection may have limited the participation of specific relevant 
population groups (e.g., disadvantaged groups such as the poor who may not be able to afford internet access) and other vulnerable groups. Therefore, the data cannot represent these groups' views, affecting the generalizability of the study findings. Another limitation of the present study was that the validation of the ESQ and its dimensions relied entirely on selfreport measures which are subject to various methods biases.

\section{Conclusion}

Despite such limitations, the Persian ESQ can be used in epidemiological research in Iran and other Persian-speaking countries to better understand the psychopathology associated with stressful situations. The findings provide evidence that the ESQ exhibits robust psychometric properties and is a valid and reliable measure of both emotional styles and the six dimensions in understanding how individuals adapt to threats to well-being.

Supplementary Information The online version contains supplementary material available at https://doi.org/10.1007/s12144-020-01205-1.

Data Availability The data that support the findings of this study are available on request from the corresponding author.

\section{Compliance with Ethical Standards}

Competing Interests The authors declared no potential conflicts of interest with respect to the research, authorship, and/or publication of this article.

Ethical Approval and Consent to Participate The study was performed in accordance with the Declaration of Helsinki and was approved and registered by the ethical and research committees from the collaborating centers. The study, including all assessments and procedures for the study, was reviewed by the National Institute for Medical Research and Development and the first author's Institutional Human Research Ethics Committee. The first author's Institutional Review Board approved the research, prospectively. All participants provided signed written consent.

\section{References}

Aldao, A. (2013). The future of emotion regulation research capturing context. Perspectives on Psychological Science, 8(2), 155-172.

Aldao, A., \& Nolen-Hoeksema, S. (2012). The influence of context on the implementation of adaptive emotion regulation strategies. Behaviour Research and Therapy, 50(7-8), 493-501. https://doi. org/10.1016/j.brat.2012.04.004.

Anderson, L. K., Claudat, K., Cusack, A., Brown, T. A., Trim, J., Rockwell, R., Nakamura, T., Gomez, L., \& Kaye, W. H. (2018). Differences in emotion regulation difficulties among adults and adolescents across eating disorder diagnoses. Journal of Clinical Psychology, 74(10), 1867-1873. https://doi.org/10.1002/jclp. 22638.

Anthony, E. K., Krysik, J., \& Kelly, C. (2019). Social-emotional wellbeing among youth living in out-of-home care. Children and Youth
Services Review, 96, 381-385. https://doi.org/10.1016/j.childyouth. 2018.12.007.

Ardelt, M., \& Grunwald, S. (2018). The importance of self-reflection and awareness for human development in hard times. Research in Human Development, 15(3-4), 187-199. https://doi.org/10.1080/ 15427609.2018.1489098.

Armstrong, S. J., van der Heijden, B. I. J. M., \& Sadler-Smith, E. (2012). Intellectual styles, management of careers, and improved work performance. In L.-F. Zhang, R. J. Sternberg, \& S. Rayner (Eds.), Handbook of intellectual styles: Preferences in cognition, learning, and thinking (pp. 273-294). New York: Springer.

Asma, S. T., \& Gabriel, R. (2019). The emotional mind: The affective roots of culture and cognition. Cambridge, MA: Harvard University Press.

Badahdah, A. M., Khamis, F., \& Mahyijari, N. A. (2020). The psychological well-being of physicians during COVID-19 outbreak in Oman. Psychiatry Research, 289, 113053. https://doi.org/10.1016/ j.psychres.2020.113053.

Barlow, D. H., Farchione, T. J., Bullis, J. R., Gallagher, M. W., MurrayLatin, H., Sauer-Zavala, S., Bentley, K. H., Thompson-Hollands, J., Conklin, L. R., Boswell, J. F., Ametaj, A., Carl, J. R., Boettcher, H. T., \& Cassiello-Robbins, C. (2017). The unified protocol for transdiagnostic treatment of emotional disorders compared with diagnosis-specific protocols for anxiety disorders. JAMA Psychiatry, 74(9), 875-884. https://doi.org/10.1001/ jamapsychiatry.2017.2164.

Barrett, L. F., \& Bliss-Moreau, E. (2009). She's emotional. He's having a bad day: Attributional explanations for emotion stereotypes. Emotion, 9(5), 649-658. https://doi.org/10.1037/a0016821.

Beaton, D. E., Bombardier, C., Guillemin, F., \& Ferraz, M. B. (2000). Guidelines for the process of cross-cultural adaptation of self-report measures. Spine, 25(24), 3186-3191. https://doi.org/10.1097/ 00007632-200012150-00014

Bloch, L., Moran, E. K., \& Kring, A. M. (2009). On the need for conceptual and definitional clarity in emotion regulation research. In A. M. Kring \& D. S. Sloan (Eds.), Emotion regulation and psychopathology (pp. 88-104). New York: Guilford.

Bryce, C., Ring, P., Ashby, S., \& Wardman, J, K. (2020). Resilience in the face of uncertainty: early lessons from the COVID-19 pandemic. Journal of Risk Research. Advance online publication. https://doi. org/10.1080/13669877.2020.1756379.

Byrne, B. M. (2016). Structural equation modeling with AMOS: Basic concepts, applications, and programming (3rd ed.). New York: Routledge.

Chen, F. F. (2007). Sensitivity of goodness of fit indexes to lack of measurement invariance. Structural Equation Modeling, 14(3), 464-504. https://doi.org/10.1080/10705510701301834.

Cheung, G. W., \& Rensvold, R. B. (2002). Evaluating goodness-of-fit indexes for testing measurement invariance. Structural Equation Modeling, 9(2), 233-255. https://doi.org/10.1207/ s15328007sem0902_5.

Chin, B., Slutsky, J., Raye, J., \& Creswell, J. D. (2019). Mindfulness training reduces stress at work: A randomized controlled trial. Mindfulness, 10(4), 627-638. https://doi.org/10.1007/s12671-0181022-0.

Cohen, J. (1992). A power primer. Psychological Bulletin, 112(1), 155 159. https://doi.org/10.1037/0033-2909.112.1.155.

Comrey, A. L., \& Lee, H. B. (2013). A first course in factor analysis (2nd ed.). Hoboken, NJ: Taylor and Francis.

Dalton, K. M., Nacewicz, B. M., Johnstone, T., Schaefer, H. S., Gernsbacher, M. A., Goldsmith, H. H., Alexander, A. L., \& Davidson, R. J. (2005). Gaze fixation and the neural circuitry of face processing in autism. Nature Neuroscience, 8, 519-526. https://doi. org/10.1038/nn1421. 
Davidson, R. J. (2015). Comment: Affective chronometry has come of age. Emotion Review, 7, 368-370. https://doi.org/10.1177/ 1754073915590844.

Davidson, R. J., \& Begley, S. (2012). The emotional life of your brain. New York, NY: Hudson Street Press.

Davidson, R. J., \& McEwen, B. S. (2012). Social influences on neuroplasticity: Stress and interventions to promote well-being. Nature Neuroscience, 15(5), 689-695. https://doi.org/10.1038/nn. 3093.

Deng, X., An, S., \& Cheng, C. (2019). Cultural differences in the implicit and explicit attitudes toward emotion regulation. Personality and Individual Differences, 149(15), 220-222. https://doi.org/10.1016/ j.paid.2019.05.057.

Dolcos, F., Katsumi, Y., Moore, M., Berggren, N., de Gelder, B., Derakshan, N., Hamm, A. O., Koster, E. H. W., Ladouceur, C. D., Okon-Singer, H., Pegna, A. J., Richter, T., Schweizer, S., van den Stock, J., Ventura-Bort, C., Weymar, M., \& Dolcos, S. (2020). Neural correlates of emotion-attention interactions: From perception, learning, and memory to social cognition, individual differences, and training interventions. Neuroscience \& Biobehavioral Reviews, 108(1), 559-601. https://doi.org/10.1016/j.neubiorev. 2019.08.017.

Every, D., McLennan, J., Reynolds, A., \& Trigg, J. (2019). Australian householders' psychological preparedness for potential natural hazard threats: An exploration of contributing factors. International Journal of Disaster Risk Reduction, 38, 101203. https://doi.org/10. 1016/j.ijdrr.2019.101203.

Ferri, F., Chiarelli, A. M., Merla, A., Gallese, V., \& Costantini, M. (2013). The body beyond the body: Expectation of a sensory event is enough to induce ownership over a fake hand. Proceedings of the Royal Society B: Biological Sciences, 280(1765). https://doi.org/10. 5061/dryad.8f251.

Finney, S. J., \& DiStefano, C. (2013). Nonnormal and categorical data in structural equation modeling (2nd ed.). In G. R. Mueller \& R. O. Hancock (Eds.), Structural equation modeling: A second course (2nd ed), (pp. 1269-314). Charlotte NC: Information age publishing.

Folkman, S. (2010). Stress, health, and coping: Synthesis, commentary, and future directions. In S. Folkman (Ed.), The Oxford handbook of stress, health, and coping. Oxford: Oxford University Press.

Folkman, S., \& Moskowitz, J. T. (2004). Coping: Pitfalls and promise. Annual Review of Psychology, 55(1), 745-774. https://doi.org/10. 1146/annurev.psych.55.090902.141456.

Ford, B. Q., Dmitrieva, J. O., Heller, D., Chentsova-Dutton, Y., Grossmann, I., Tamir, M., Uchida, Y., Koopmann-Holm, B., Floerke, V. A., Uhrig, M., Bokhan, T., \& Mauss, I. B. (2015). Culture shapes whether the pursuit of happiness predicts higher or lower well-being. Journal of Experimental Psychology. General, 144(6), 1053-1062. https://doi.org/10.1037/xge0000108.

Fredrickson, B. L., \& Joiner, T. (2018). Reflections on positive emotions and upward spirals. Perspectives on Psychological Science, 13(2), 194-199. https://doi.org/10.1177/1745691617692106.

Fredrickson, B. L., Tugade, M. M., Waugh, C. E., \& Larkin, G. R. (2003). What good are positive emotions in crises? A prospective study of resilience and emotions following the terrorist attacks on the United States on September 11th, 2001. Journal of Personality and Social Psychology, 84(2), 365-376. https://doi.org/10.1037// 0022-3514.84.2.365.

Gratz, K. L., \& Roemer, L. (2004). Multidimensional assessment of emotion regulation and dysregulation: Development, factor structure, and initial validation of the difficulties in emotion regulation scale. Journal of Psychopathology and Behavioral Assessment, 26(1), 4154. https://doi.org/10.1023/B:JOBA.0000007455.08539.94.

Gross, J. J., \& Jazaieri, H. (2014). Emotion, emotion regulation, and psychopathology: An affective science perspective. Clinical
Psychological Science, 2(4), 387-401. https://doi.org/10.1177/ 2167702614536164.

Gross, J. J., \& John, O. P. (2003). Individual differences in two emotion regulation processes: Implications for affect, relationships, and wellbeing. Journal of Personality and Social Psychology, 85(2), 348362. https://doi.org/10.1037/0022-3514.85.2.348.

Gross, J. J., Uusberg, H., \& Uusberg, A. (2019). Mental illness and wellbeing: An affect regulation perspective. World Psychiatry, 18(2), 130-139. https://doi.org/10.1002/wps.20618.

Hagerty, S. L., \& Williams, L. M. (2020). The impact of COVID-19 on mental health: The interactive roles of brain biotypes and human connection. Brain, Behavior, \& Immunity - Health, 5(3), 100078. https://doi.org/10.1016/j.bbih.2020.100078.

Helmreich, I., Kunzler, A., Chmitorz, A., König, J., Binder, H., Wessa, M., \& Lieb, K. (2017). Psychological interventions for resilience enhancement in adults. The Cochrane Database of Systematic Reviews, 2017(2), CD012527. https://doi.org/10.1002/14651858. CD012527.

Henseler, J., Hubona, G., \& Ray, P. A. (2016). Using PLS path modeling in new technology research: Updated guidelines. Industrial Management \& Data Systems, 116(1), 2-20. https://doi.org/10. 1108/imds-09-2015-0382.

Holmes, E. A., O’Connor, R. C., Perry, V. H., Tracey, I., Wessely, S., Arseneault, L., et al. (2020). Multidisciplinary research priorities for the COVID-19 pandemic: A call for action for mental health science. The Lancet Psychiatry, 7(6), 547-560. https://doi.org/10. 1016/s2215-0366(20)30168-1.

Hu, L. T., \& Bentler, P. M. (1999). Cutoff criteria for fit indexes in covariance structure analysis: Conventional criteria versus new alternatives. Structural Equation Modeling, 6(1), 1-55. https://doi. org/10.1080/10705519909540118.

Izard, C. E. (2010). The many meanings/aspects of emotion: Definitions, functions, activation, and regulation. Emotion Review, 2(4), 363370. https://doi.org/10.1177/1754073910374661.

Jobson, L., Mirabolfathi, V., Moshirpanahi, S., Parhoon, H., Gillard, J., Mukhtar, F., Moradi, A. R., \& Mohan, S. N. (2019). Investigating emotion in Malay, Australian and Iranian individuals with and without depression. Scientific Reports, 9, 18344. https://doi.org/10.1038/ s41598-019-54775-x.

Kahneman, D., \& Deaton, A. (2010). High income improves evaluation of life but not emotional well-being. Proceedings of the National Academy of Sciences, 107(38), 16489-16493. https://doi.org/10. 1073/pnas.1011492107.

Kesebir, P., Gasiorowska, A., Goldman, R., Hirshberg, M. J., \& Davidson, R. J. (2019). Emotional style questionnaire: A multidimensional measure of healthy emotionality. Psychological Assessment, 31(10), 1234-1246. https://doi.org/10.1037/ pas0000745.

Killingsworth, M. A., \& Gilbert, D. T. (2010). A wandering mind is an unhappy mind. Science, 330, 932. https://doi.org/10.1126/science. 1192439.

Kim, M. J., \& Whalen, P. J. (2009). The structural integrity of an amygdalaprefrontal pathway predicts trait anxiety. The Journal of Neuroscience, 29, 11614-11618. https://doi.org/10.1523/ JNEUROSCI.2335-09.2009.

Kline, R. B. (2016). Principles and practices of structural equation modelling. In T. D. Little (Ed.), Methodology in the social sciences (4th ed.). New York: Guilford Press.

Kong, F., Yang, K., Sajjad, S., Yan, W., Li, X., \& Zhao, J. (2019). Neural correlates of social well-being: Gray matter density in the orbitofrontal cortex predicts social well-being in emerging adulthood. Social Cognitive and Affective Neuroscience, 14(3), 319327. https://doi.org/10.1093/scan/nsz008.

Kret, M. E., Sinke, C. B. A., \& de Gelder, B. (2011). Emotion perception and health. In I. Nyklíček et al. (Eds.), Emotion regulation and well- 
being (pp. 261-280). New York: Springer. https://doi.org/10.1007/ 978-1-4419-6953-8 16.

Laird, K. T., Krause, B., Funes, C., \& Lavretsky, H. (2019). Psychobiological factors of resilience and depression in late life. Translational Psychiatry, 9(1), 1-18. https://doi.org/10.1038/ s41398-019-0424-7.

Lapate, R. C., \& Heller, A. S. (2020). Context matters for affective chronometry. Nature Human Behaviour, 5, 1-2. https://doi.org/10.1038/ s41562-020-0860-7.

Lapate, R. C., van Reekum, C. M., Schaefer, S. M., Greischar, L. L., Norris, C. J., Bachhuber, D. R., Ryff, C. D., \& Davidson, R. J. (2014). Prolonged marital stress is associated with short-lived responses to positive stimuli. Psychophysiology, 51(6), 499-509. https://doi.org/10.1111/psyp.12203.

Lavender, J. M., Wonderlich, S. A., Engel, S. G., Gordon, K. H., Kaye, W. H., \& Mitchell, J. E. (2015). Dimensions of emotion dysregulation in anorexia nervosa and bulimia nervosa: A conceptual review of the empirical literature. Clinical Psychology Review, 40, 111122. https://doi.org/10.1016/j.cpr.2015.05.01022

Leahy, R. L., Tirch, D. D., \& Napolitano, L. A. (2011). Emotion regulation in psychotherapy: A practitioner's guide. New York: Guilford Press.

Lemola, S., Räikkönen, K., Gomez, V., \& Allemand, M. (2013). Optimism and self-esteem are related to sleep. Results from a large community-based sample. International Journal of Behavioral Medicine, 20(4), 567-571. https://doi.org/10.1007/s12529-0129272-z.

Li, J., Ghosh, R., \& Nachmias, S. (2020). In a time of COVID-19 pandemic, stay healthy, connected, productive, and learning: Words from the editorial team of HRDI. Human Resource Development International, 23(3), 199-207. https://doi.org/10.1080/13678868. 2020.1752493.

Limcaoco, R, S, G., Mateos, E, M., Fernandez, J, M., \& Roncero, C. (2020). Anxiety, worry and perceived stress in the world due to the COVID-19 pandemic, march 2020. Preliminary results. medRxiv. https://doi.org/10.1101/2020.04.03.20043992.

Linnenbrink-Garcia, L., \& Pekrun, R. (2011). Students' emotions and academic engagement: Introduction to the special issue. Contemporary Educational Psychology, 36(1), 1-3. https://doi. org/10.1016/j.cedpsych.2010.11.004.

Lobue, V., Pérez-Edgar, K., \& Buss, K. A. (2019). Handbook of emotional development. Cham: Springer. https://doi.org/10.1007/978-3030-17332-6.

Lutz, A., Slagter, H. A., Dunne, J. D., \& Davidson, R. J. (2008). Attention regulation and monitoring in meditation. Trends in Cognitive Sciences, 12(4), 163-169.

Margolis, S., \& Lyubomirsky, S. (2018). Cognitive outlooks and wellbeing. In E. Diener, S. Oishi, \& L. Tay (Eds.), Handbook of wellbeing (pp. 143-158). Salt Lake City: DEF Publishers.

Mesquita, B., De Leersnyder, J., \& Albert, D. (2014). The cultural regulation of emotions. In J. J. Gross (Ed.), Handbook of emotion regulation (pp. 284-301). New York: Guilford Press.

Millstein, R. A., Celano, C. M., Beale, E. E., Beach, S. R., Suarez, L., Belcher, A. M., Januzzi, J. L., \& Huffman, J. C. (2016). The effects of optimism and gratitude on adherence, functioning and mental health following an acute coronary syndrome. General Hospital Psychiatry, 43, 17-22. https://doi.org/10.1016/j.genhosppsych. 2016.08.006.

Mrazek, M. D., Franklin, M. S., Phillips, D. T., Baird, B., \& Schooler, J. W. (2013). Mindfulness training improves working memory capacity and GRE performance while reducing mind wandering. Psychological Science, 24, 776-781.

Myruski, S., Bonanno, G. A., Gulyayeva, O., Egan, L. J., \& DennisTiwary, T. A. (2017). Neurocognitive assessment of emotional context sensitivity. Cognitive, Affective, \& Behavioral Neuroscience, 17(5), 1058-1071. https://doi.org/10.3758/s13415-017-0533-9.
Nazari, N., Aligholipour, A., Sadeghi, M. (2020). Transdiagnostic treatment of emotional disorders for women with multiple sclerosis: a randomized controlled trial. BMC Women's Health, 20(1), 245. https://doi.org/10.1186/s12905-020-01109-z.

Norman, E., \& Price, M. C. (2012). Social intuition as a form of implicit learning: Sequences of body movements are learned less explicitly than letter sequences. Advances in Cognitive Psychology, 8(2), 121131. https://doi.org/10.2478/v10053-008-0109-x.

Norman, S. B., Cissell, S. H., Means-Christensen, A. J., \& Stein, M. B. (2006). Development and validation of an overall anxiety severity and impairment scale (OASIS). Depression and Anxiety, 23(4), 245-249. https://doi.org/10.1002/da.20182.

Norton, P. J., \& Paulus, D. J. (2016). Toward a unified treatment for emotional disorders: Update on the science and practice. Behavior Therapy, 47(6), 854-868. https://doi.org/10.1016/j.beth.2015.07. 002.

Ong, E., \& Thompson, C. (2019). The importance of coping and emotion regulation in the occurrence of suicidal behavior. Psychological Reports, 122(4), 1192-1210. https://doi.org/10.1177/ 0033294118781855

Oser, M., Khan, A., Kolodziej, M., Gruner, G., Barsky, A, J., \& Epstein, L. (2019). Mindfulness and interoceptive exposure therapy for anxiety sensitivity in atrial fibrillation: A pilot study. Behavior Modification. Advance online publication. https://doi.org/10.1177/ $0145445519877619,014544551987761$.

Ostir, G. V., Markides, K. S., Black, S. A., \& Goodwin, J. S. (2000). Emotional well-being predicts subsequent functional Independence and survival. Journal of the American Geriatrics Society, 48(5), 473-478. https://doi.org/10.1111/j.1532-5415.2000.tb04991.x.

Phillips, W. J. (2018). Future-outlook mediates the association between self-compassion and well-being. Personality and Individual Differences, 135, 143-148. https://doi.org/10.1016/j.paid.2018.07. 006.

Pressman, S. D., Jenkins, B. N., \& Moskowitz, J. T. (2019). Positive affect and health: What do we know and where next should we go? Annual Review of Psychology, 70(1), 627-650. https://doi.org/ 10.1146/annurev-psych-010418-102955.

Renna, M. E., Quintero, J. M., Fresco, D. M., \& Mennin, D. S. (2017). Emotion regulation therapy: A mechanism-targeted treatment for disorders of distress. Frontiers in Psychology, 8, 98. https://doi. org/10.3389/fpsyg.2017.00098.

Restubog, S. L. D., Ocampo, A. C. G., \& Wang, L. (2020). Taking control amidst the chaos: Emotion regulation during the COVID19 pandemic. Journal of Vocational Behavior, 103440, 103440. https://doi.org/10.1016/j.jvb.2020.103440.

Ricard, M., Lutz, A., \& Davidson, R. J. (2014). Mind of the meditator. Scientific American, 311(5), 38-45. https://doi.org/10.1038/ scientificamerican1114-38.

Rosellini, A. J., \& Brown, T. A. (2019). The multidimensional emotional disorder inventory (MEDI): Assessing transdiagnostic dimensions to validate a profile approach to emotional disorder classification. Psychological Assessment, 31(1), 59-72. https://doi.org/10.1037/ pas0000649.

Roudini, J., Khankeh, H. R., \& Witruk, E. (2017). Disaster mental health preparedness in the community: A systematic review study. Health Psychology Open, 4(1), 1-12. https://doi.org/10.1177/ 2055102917711307.

Sander, D. (2013). Models of emotion: The affective neuroscience approach. In J. L. Armony \& P. Vuilleumier (Eds.), The Cambridge handbook of human affective neuroscience (pp.5-53). Cambridge: Cambridge University press.

Santarone, K., McKenney, M., \& Elkbuli, A. (2020). Preserving mental health and resilience in frontline healthcare workers during COVID19. American Journal of Emergency Medicine, S0735-6757(20) 30258-8. https://doi.org/10.1016/j.ajem.2020.04.030, 1531. 
Scal, P., Ireland, M., \& Borowsky, I. W. (2003). Smoking among American adolescents: A risk and protective factor analysis. Journal of Community Health, 28(2), 79-97. https://doi.org/10. 1023/a:1022691212793.

Schumacker, R. E., \& Lomax, R. G. (2016). A beginner's guide to structural equation modeling (4th ed.). New York: Routledge. https://doi. org/10.4324/9780203851319.

Schuyler, B. S., Kral, T. R., Jacquart, J., Burghy, C. A., Weng, H. Y., Perlman, D. M., Bachhuber, D. R., Rosenkranz, M. A., Maccoon, D. G., van Reekum, C. M., Lutz, A., \& Davidson, R. J. (2014). Temporal dynamics of emotional responding: Amygdala recovery predicts emotional traits. Social Cognitive and Affective Neuroscience, 9(2), 176-181. https://doi.org/10.1093/scan/nss131.

Shackman, A. J., Fox, A. S., Lapate, R. C., \& Davidson, R. J. (2018). Introduction. In A. S. Fox, A. J. Shackman, R. C. Lapate, \& R. J. Davidson (Eds.), The nature of emotion: Fundamental questions (2nd ed.). (pp. xxiii-xxiv). New York: Oxford University press.

Skevington, S. M., Sartorius, N., Amir, M., Sartorius, N., Orley, J., Kuyken, W., et al. (2004). Developing methods for assessing quality of life in different cultural settings - the history of the WHOQOL instruments. Social Psychiatry and Psychiatric Epidemiology, 39(1), 1-8. https://doi.org/10.1007/s00127-004-0700-5.

Smith, J. L., \& Hanni, A. A. (2019). Effects of a savoring intervention on resilience and well-being of older adults. Journal of Applied Gerontology, 38(1), 137-152. https://doi.org/10.1177/ 0733464817693375.

Steinhauser, R., Maier, M. E., \& Steinhauser, M. (2017). Neural signatures of adaptive post-error adjustments in visual search. NeuroImage, 150, 270-278. https://doi.org/10.1016/j.neuroimage. 2017.02.059.

Tolin, D. F., \& Foa, E. B. (2008). Sex differences in trauma and posttraumatic stress disorder: A quantitative review of 25 years of research. Psychological Trauma: Theory, Research, Practice, and Policy, S(1), 37-85. https://doi.org/10.1037/1942-9681.s.1.37.
Tsai, J. L., \& Qu, Y. (2018). The promise of neuroscience for understanding the cultural shaping of emotion and other feelings. Culture and Brain, 6(2), 99-101. https://doi.org/10.1007/s40167-018-0069-z.

van Kleef, G. A., Cheshin, A., Fischer, A. H., \& Schneider, I. K. (2016). Editorial: The social nature of emotions. Frontiers in Psychology, 7, 896. https://doi.org/10.3389/fpsyg.2016.00896.

Vandenbos, G. R. (2015). APA dictionary of psychology. (2nd ed.) American Psychological Association. https://doi.org/10.1037/ 14646-000.

Wang, K., Goldenberg, A., Dorison, C., Miller, J., Lerner, J., Gross, J. \& Psychological Science Accelerator. (2020). A global test of brief reappraisal interventions on emotions during the COVID-19 pandemic. Leibniz Institut für Psychologische Information und Dokumentation (ZPID). https://doi.org/10.23668/ PSYCHARCHIVES.2957.

Watson, D., Clark, L. A., \& Tellegen, A. (1988). Development and validation of brief measures of positive and negative affect: The PANAS scales. Journal of Personality and Social Psychology, 54(6), 1063-1070. https://doi.org/10.1037//0022-3514.54.6.1063.

WHO QOL Group (1998). Development of the World Health Organization WHO Quality of Life Assessment. Psychological Medicine, 28(3), 551-558 10.5.12.

Wilner Tirpak, J., Cassiello-Robbins, C., Ametaj, A., Olesnycky, O. S., Sauer-Zavala, S., Farchione, T. J., \& Barlow, D. H. (2019). Changes in positive affect in cognitive-behavioral treatment of anxiety disorders. General Hospital Psychiatry, 61, 111-115. https://doi.org/10. 1016/j.genhosppsych.2019.06.008.

Yang, H., \& Ma, J. (2020). How an epidemic outbreak impacts happiness: Factors that worsen (vs. protect) emotional well-being during the coronavirus pandemic. Psychiatry Research, 289, 113045. https://doi.org/10.1016/j.psychres.2020.113045.

Publisher's Note Springer Nature remains neutral with regard to jurisdictional claims in published maps and institutional affiliations. 